\title{
Exposición toxicológica en las grandes alturas: ¿es necesario corregir los valores límite umbral de exposición de tóxicos?
}

\author{
Toxicology exposure at high altitude: is it necessary to correct toxic
} exposure threshold limit values?

\author{
Augusto V. Ramírez \\ American College of Occupational and Environmental Medicine.
}

\begin{abstract}
Resumen
El nativo de altura posee variantes anatomofisiológicas en el sistema respiratorio. Las de mayor significado son los aumentos de amplitud torácica, ventilación pulmonar, difusión alveolo/capilar y del contenido arterial de oxígeno. En salud ocupacional, para controlar los riesgos del trabajo se aplica valores limites de exposición desarrollados a nivel del mar y para poblaciones con características físicas y laborales diferentes a las de altura. Fisiológicamente, un trabajo seguro en la altura se caracteriza porque el organismo no demanda mayor energía que a nivel del mar para realizarlo, a pesar de la caída de la presión barométrica y esto es así hasta los 2500 msnm. De acuerdo a esta premisa, cuando por el nivel de altitud el organismo compensa la baja presión barométrica con aumento de la ventilación pulmonar, es necesario corregir los límites de exposición. Aún no se ha establecido límites de exposición seguros en la altura, lo que implica continuar investigando.
\end{abstract}

Palabras clave: Fisiologia respiratoria; hombre de la altura; toxicología ocupacional; corrección límites exposición.

\section{Abstract}

In occupational health to control risk magnitude we apply threshold limit values. Man at high altitude has anatomical-physiological variants in his respiratory system; most important are increases in thoracic capacity, pulmonary ventilation, alveolar/capillary diffusion, and arterial oxygen content. Physiological safe work at high altitude consists in the organism not using extra energy to compensate barometric pressure fall. In respiratory occupational toxicology we must initiate correction when ventilatory response to hypobaric hypoxia becomes unsafe and this occurs at 8000 feet above sea level. Accordingly we should correct exposure limits value applying to work at high altitude. In order to establish safe parameters of toxicological exposition at high altitude it is necessary to investigate these aspects in high altitude workers.

Key words: Respiratory physiology; man at high altitude; occupational toxicology; exposure's limits correction.

\section{An Fac med. 2011;72(1):61-7}

\section{INTRODUCCIÓN}

Responder la pregunta titular implica, primero, establecer qué se entiende en fisiología respiratoria por 'gran altura' y luego revisar los estudios actuales en fisiología de la altura y compararlos con los de nivel del mar, para sopesar si acaso son diferentes. También, se ha de dilucidar si el habitante del altiplano posee características físicas y fisiológicas que le permitan aclimatarse y lo pongan en condición de realizar cualquier trabajo físico o mental a esos niveles de altura.

\section{GRAN ALTURA}

Los fisiólogos puros han determinado que la cota desde la cual se debe considerar 'altura' es 3000 metros sobre el nivel del mar (msnm), pues a partir de allí, entre otras razones, la presión parcial de oxígeno (PO2) en el aire tra- queal comienza a disminuir a menos de 100 torrelios (torr), nivel crítico en la cascada de oxígeno. Consideran 'gran altura' a partir de $3600 \mathrm{msnm}$.

Fisiológicamente, los niveles de altitud se definen según la siguiente relación establecida por el Lake Louise Consensus ${ }^{(1)}$.

\begin{tabular}{ccc} 
Denominación & Altura metros snm & Altura pies snm \\
Altura & $3000-3658$ & $10000-12000$ \\
Gran altura & $3658-5487$ & $12000-18000$ \\
Altura extrema & $>5487$ & $>18000$ \\
\hline
\end{tabular}

$\mathrm{snm}=$ sobre el nivel del $\mathrm{mar}$. 
En fisiología del trabajo, los investigadores sostienen que no existe diferencia para el desempeño físico entre nivel del mar y la altura, hasta un nivel de $2500 \mathrm{msnm}$. En cambio, a partir de esa altura, la capacidad de realizar un trabajo intenso se reduce claramente ${ }^{(2)}$. Por este motivo fisiológico, en Salud Ocupacional, el concepto de altitud para trabajo seguro se define como aquel en que el organismo aún compensa sin mayor demanda de energía la caída de la presión barométrica y, por tanto, cualquier trabajo físico que se realice hasta esos niveles de altitud no requiere consumo extra de energía.

En toxicología ocupacional respiratoria de la altura, se considera como punto crítico aquel nivel a partir del cual la respuesta ventilatoria a la hi- poxia hipobárica implica consumo extra de energía, con el consiguiente aumento del riesgo. Esto es así desde los $2500 \mathrm{msnm}$ (8000 pies sobre el nivel del mar [psnm]), pues si bien los estudios fisiológicos puros definen el nivel crítico de respuesta compensatoria en $3000 \mathrm{msnm}^{(1-4)}$, la mayor parte de ellos, sino todos, han sido realizados en condiciones de hipoxia aguda y con aire sin contenido tóxico. Lo que sucede ante una mayor demanda de energía en periodos prolongados de exposición a tóxicos en meses o años aún no ha sido suficientemente dilucidado ${ }^{(5)}$.

Para términos de la presente revisión y según lo expuesto, 'gran altura' es todo aquel lugar geográfico que se halle ubicado más allá de los 3658 msnm (12000 psnm).

\section{FISIOLOGÍA EN LAS GRANDES ALTURAS Y A NIVEL DEL MAR}

En cuanto a verificar si la fisiología humana en esas altitudes es diferente a la de nivel del mar, la investigación sobre aclimatización del hombre a la altura es amplia y abarca estudios en habitantes de diferentes grandes alturas ubicadas, entre otros lugares, en el Tibet -Everest-, en los montes Blanco -Alpes-y McKinley -Alaska-, seres humanos que, por cierto, tienen mecanismos fisiológicos compensatorios propios.

En ese contexto, Chiodi, en 1957, halló aumento de 39\% en la ventilación pulmonar de no aclimatados recién llegados desde el nivel del mar, a $3990 \mathrm{~m}$, y de $47 \%$ si subían a $4515 \mathrm{~m}$. El estudio incluyó a los ya aclimatados a alturas

Tabla 1. Algunas variantes de aclimatación del hombre en la altura.

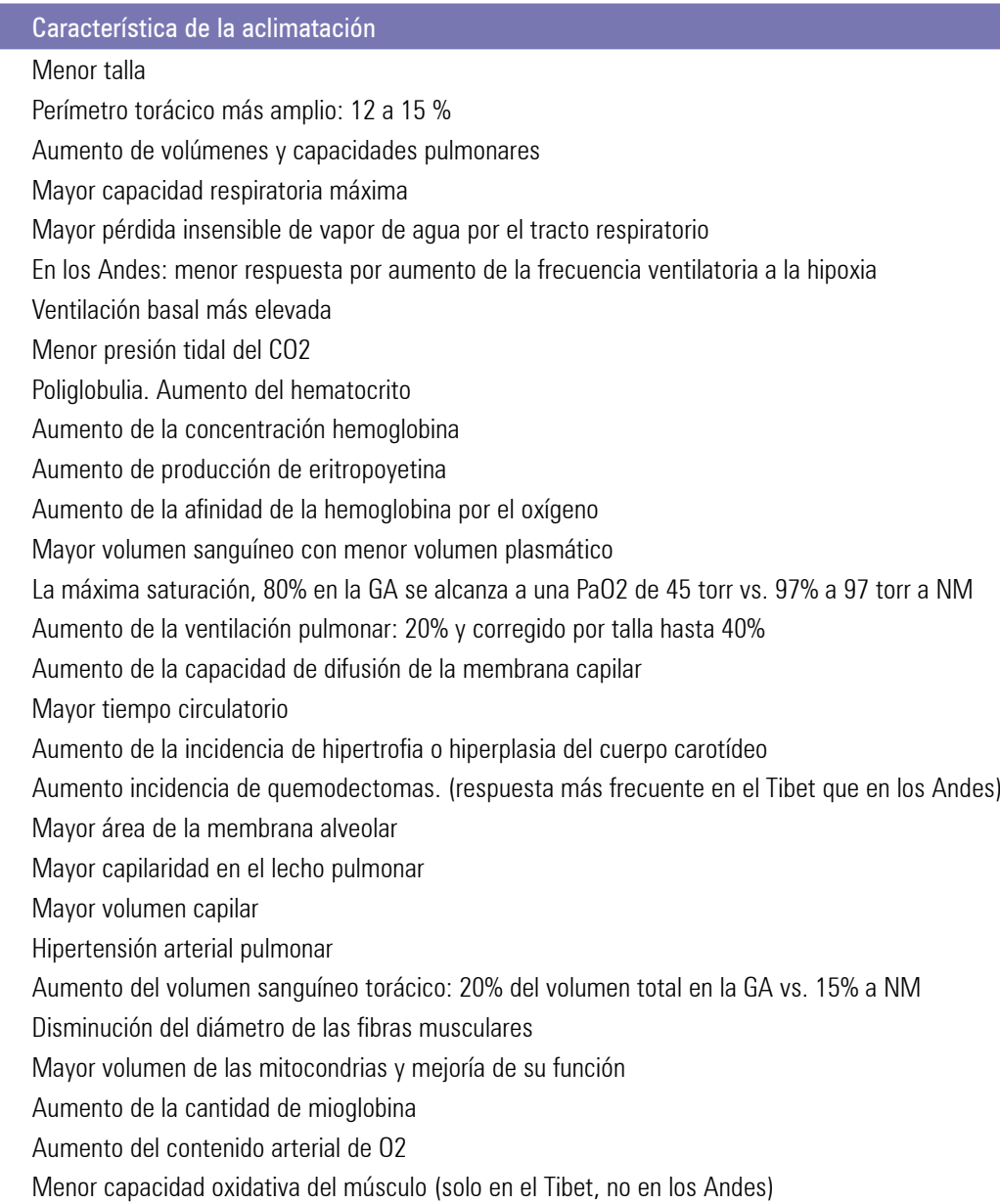




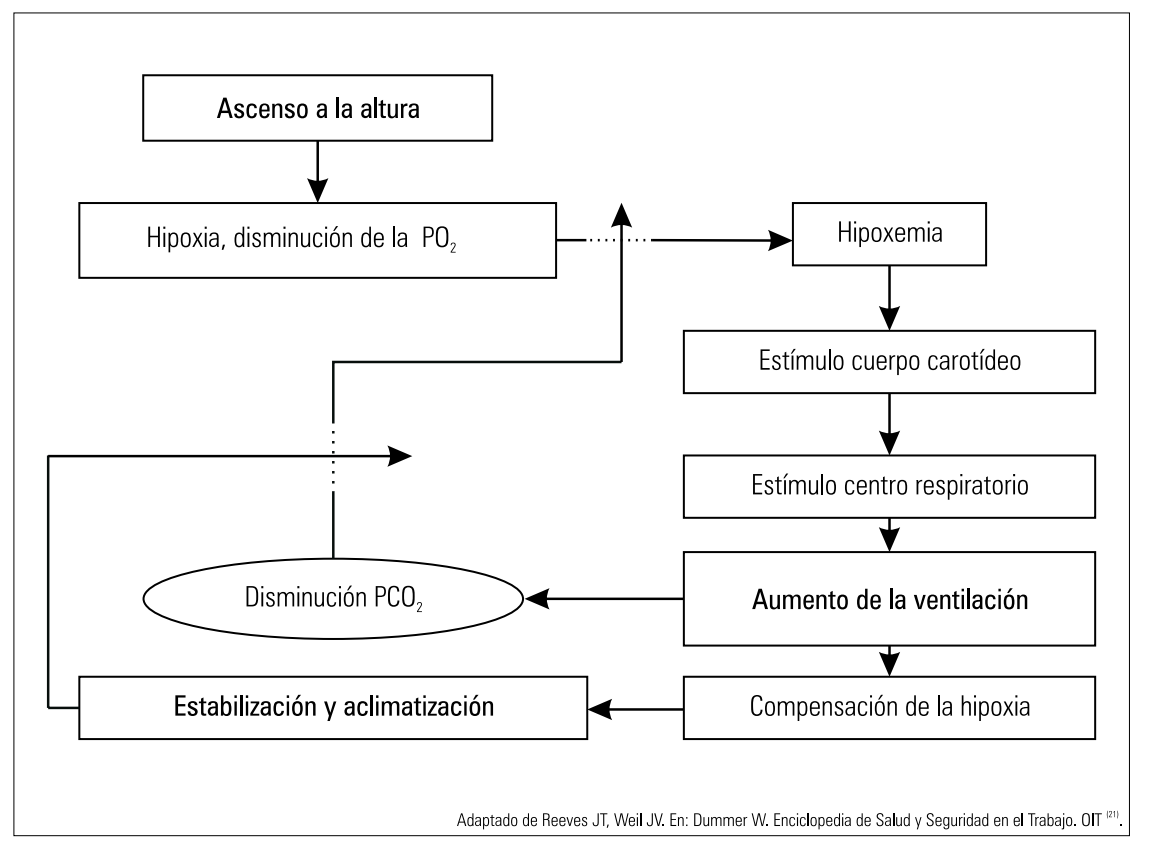

Figura 1. Mecanismo de aclimatación en ascenso agudo a la altura.

superiores a $4990 \mathrm{msnm}$, quienes también incrementaron su ventilación hasta en $29 \%{ }^{(4)}$.

En nuestros Andes, investigadores peruanos han realizado estudios profundos sobre aclimatización del hombre a la altura. Estudios pioneros son los de Monge Medrano, Hurtado, Velásquez, Rotta y actuales los de Marticorena, Monge Casinelli, Cruz Jibaja, León-Velarde, Gamboa y otros muchos, todos ellos encaminados a conocer la fisiología del hombre de la altura, principalmente en la sierra central del Perú, tanto en condiciones de laboratorio como de campo ${ }^{(6)}$.

Frisancho, en Puno (7), y la escuela boliviana ${ }^{(8)}$ tienen similar experiencia con el andino de ancestro quechuaaimara o aimara.

La tabla 1 resume algunos de los aspectos fisiológicos y anatómicos sobresalientes en la aclimatización del hombre a la altura condicionados por la hipoxia hipobárica. De ellos, los de mayor significado respiratorio son el aumento de ventilación pulmonar, difusión alvéolo/ capilar, contenido arterial de $\mathrm{O} 2$ y de capacidad torácica, modificaciones que han devenido en necesarias para vivir a esos niveles de altitud. Los investigadores han determinado que esos cambios dependen de mecanismos cuasi genéticos de aclimatización (fenotípicos), lo que hace al nativo de la gran altura mejor dotado para residir allí que el llanero recién llegado o el costeño residente en la altura ${ }^{(9-13)}$.

\section{Hipoxia hipobárica}

Etimológicamente, hipoxemia es la disminución del oxígeno en la sangre causada por la hipoxia, que es la disminución del oxígeno en el aire; en este caso, y por ser causada por la disminución de la presión barométrica (PB), se la llama hipoxia hipobárica ${ }^{(3)}$. Se ha determinado que problemas médicos y enfermedades relacionadas con la altura se presentan ya entre los 1500 y $2000 \mathrm{msnm}$, pero son más frecuentes entre los 2400 y $4300^{(14)}$.

La tabla 2 presenta los valores de presión atmosférica en torrelios por nivel de altitud, otra columna muestra la $\mathrm{PO} 2$ en el aire ambiental y una tercera la $\mathrm{PO} 2$ del aire traqueal, donde el oxígeno, humedecido y atemperado, recién está listo para ir al intercambio gaseoso en pulmones. De acuerdo a esas magnitudes, la PB a 3000 msnm es el 70\% de la de nivel del mar, a 4000 el $60 \%$ y a 5500 el $50 \%$.

De otro lado, la exposición aguda a grandes alturas desencadena mecanismos fisiológicos 'de urgencia' para lograr el mismo objetivo en el menor tiempo posible. Estos son mostrados en la figura 1 .

\section{ADAPTACIÓN DEL HOMBRE NATIVO EN LAS GRANDES ALTURAS}

\section{El hombre del ande}

Estudios realizados desde fines del s. XIX en el nativo del ande peruano demuestran que posee mayor perímetro torácico y capacidad pulmonar, así como menor talla que el hombre del llano. Las explicaciones aceptadas para estas diferencias indican que son respuestas de aclimatización: la menor talla se relaciona con maduración retrasada; el aumento del perímetro del tórax y de la capacidad pulmonar resultan de la adaptación funcional a la difícil respiración en la gran altura andina, mecanismos por cierto diferentes del nativo de otras grandes alturas, como las del Himalaya, donde la corrección va más a la compensación por aumento de la frecuencia respiratoria. Debido a esta circunstancia, el Homo sapiens andino tiene un modelo somático diferente al del llano, pues por la necesidad de residir en la altura ha desarrollado características propias que le permiten vivir en esas condiciones ambientales particulares y ha debido elegir entre modificar el metabolismo celular para que sus células vivan en condiciones hipóxicas -hecho improbable a esta altura evolutiva- o, lo más fisiológico, aumentar la profundidad de su ventilación para modificar la conducción del oxígeno en la cascada.

Por los estudios realizados, se sabe que las variantes anatómicas y fisiológicas respiratorias decisivas para ese fin son: el aumento de entre 12 y $15 \%$ del perímetro torácico y de $20 \%$ en su capacidad vital forzada, la que, al corregirse por talla, llega a $40 \%{ }^{(6,9,15-17)}$. 
Tabla 2. Presión atmosférica, $\mathrm{PO}_{2}$ y $\mathrm{PO}_{2}$ traqueal, por nivel de altitud.

\begin{tabular}{|c|c|c|c|}
\hline Altura msnm & $\begin{array}{l}\text { Presión atmosférica } \\
\text { Torr }\end{array}$ & $\begin{array}{l}\mathrm{PO}_{2} \text { por nivel de altitud } \\
\text { Torr }\end{array}$ & $\begin{array}{l}\mathrm{PO}_{2} \text { en aire traqueal, por altitud } \\
\text { Torr }\end{array}$ \\
\hline 0 & 760 & 159,61 & 149 \\
\hline 500 & 716 & 150,36 & 140 \\
\hline 1000 & 674 & 141,54 & 131 \\
\hline 1500 & 634 & 133,14 & 123 \\
\hline 2000 & 596 & 125,16 & 115 \\
\hline 2500 & 560 & 117,61 & 107 \\
\hline 3000 & 526 & 110,25 & 100 \\
\hline 3500 & 493 & 103,53 & 93 \\
\hline 4000 & 462 & 97,02 & 87 \\
\hline 4500 & 433 & 90,93 & 81 \\
\hline 5000 & 405 & 85,05 & 75 \\
\hline 5500 & 379 & 79,59 & 69 \\
\hline 6000 & 354 & 74,34 & 64 \\
\hline 7000 & 308 & 64,68 & 55 \\
\hline 8000 & 267 & 56,07 & 46 \\
\hline 9000 & 230 & 48,51 & 38 \\
\hline 10000 & 198 & 41,58 & 32 \\
\hline
\end{tabular}

\section{Condiciones de trabajo en la altura}

En la altura de nuestros andes, y más allá de los $3500 \mathrm{msnm}$, vive alrededor de $15 \%$ de los peruanos, ocupados principalmente -en forma directa o indirecta- en minero metalurgia extractiva primaria. La minería legal, actividad característica del trabajo en las grandes alturas del Perú, comúnmente se localiza por encima de ese nivel y, salvo una agricultura y ganadería cuasi artesanales y rudimentarias, no existe otra actividad laboral tan tecnificada y amplia $^{(14,18)}$.

Las condiciones de trabajo en general y de los mineros peruanos en particular han cambiado radicalmente en los últimos 20 años, incluyendo los horarios y el trabajo por turnos, tanto que ahora el lugar de residencia de los trabajadores se encuentra en sitios alejados de la labor minera, casi siempre a menor altitud, y algunas veces a nivel del mar, lo cual condiciona acudir diariamente a trabajar en zonas de altura, hecho que a su vez conlleva rápidas e intensas variaciones fisiológicas en su sistema cardiorrespiratorio por ese frecuente ascenso y descenso ${ }^{(18,19)}$.
En adición a ese estrés fisiológico, conforme se asciende aparecen diferentes condiciones ambientales que contribuyen a hacer más laborioso el trabajo en la gran altura: baja progresiva de la temperatura, disminución de la densidad del aire, disminución de la humedad, aumento de la radiación cósmica, disminución de la gravedad y otros. Estos cambios generan, entre otros, alteraciones en el ritmo circadiano, estrés y fatiga, sin menospreciar las diferencias en el comportamiento toxicológico de los agresores, los que impactan en la salud del trabajador por mayor tiempo de exposición y menor tiempo de detoxificación $^{(5,19)}$.

Las condiciones de trabajo de la minero-metalurgia a esos niveles tienen pues implicancias que van del diferente comportamiento físico-químico de los tóxicos a mayor tiempo de labor continua ${ }^{(18)}$.

\section{Tóxicos respiratorios: valores límite umbral de exposición (TLV) y altura}

En el aire, la concentración de un químico se mide en partes por millón (ppm) o en peso por metro cúbico $\left(\mathrm{mg} / \mathrm{m}^{3}\right)$. La relación de las moléculas de un tóxico con las moléculas del oxígeno no cambia por las variaciones en la presión barométrica. Por tanto, su concentración en un volumen fijo de aire inspirado tendrá siempre el mismo efecto biológico a nivel del mar que en la altura. Por ejemplo, ventilar 100 litros de aire por minuto que contenga una concentración de vapores de mercurio de 0,02 $\mathrm{mg} / \mathrm{m}^{3}$ a nivel del mar tendrá el mismo efecto tóxico que respirarlo a 4000 msnm ${ }^{(19,20)}$. Sin embargo, debido a la disminución de la presión barométrica existe un incremento compensatorio de la ventilación pulmonar con respiraciones más profundas que, aunadas a la mayor capacidad del tórax, condicionan a que, en la altura, el volumen de aire ventilado en unidad de tiempo sea mayor que en el llano. Así, a nivel del mar, donde $\mathrm{PO} 2$ traqueal es de 149 torr, desempeñar un trabajo físico estándar [que consuma p.ej. 4 litros/min promedio de oxígeno] requiere ventilar $105 \mathrm{~L} /$ min, mientras que para el mismo trabajo a $3000 \mathrm{msnm}-\mathrm{PO} 2$ traqueal de 100 torr-, el requerimiento es $190 \mathrm{~L} / \mathrm{min}^{(2)}$. Por tanto, si medimos el tóxico en peso, la dosis que ingrese en la misma unidad de tiempo será mayor ${ }^{(20-22)}$.

$\mathrm{Al}$ aplicar valores límite umbral de exposición en salud ocupacional - $T L V^{\mathrm{R}}$, $\mathrm{ACGIH}^{\mathrm{R}}$, por sus siglas inglesas ${ }^{(23)}$-, es fundamental tener en cuenta los mecanismos de aclimatización, porque esos valores u otros similares no proponen recomendación alguna para ajustarlos a la gran altura. La fisiología respiratoria a esos niveles evidencia que existe, entre otros, un importante incremento de la ventilación pulmonar y, por ende, para reducir el riesgo de daño a la salud resultante de esas condiciones de trabajo propias de la altitud, es necesario hacer correcciones o en todo caso ajustar el tiempo de exposición.

Otro hecho importante a considerar cuando se realice una corrección es que esos valores, vigentes en países desarrollados, se les define asumiendo una jornada laboral de 8 horas para una se- 
mana de 5 días o 40 horas/semana. En nuestros andes, la aplicación técnica y realista de los TLV pasa por corregirlos primero, por tiempo de labor de 40 a 48 horas/semana, para luego llevarlos a los valores reales que condiciona la fisiología en la gran altura; y solo entonces el TLV ambiental será seguro para el trabajador ${ }^{(21-23)}$. En uno u otro caso, la pregunta específica es cómo y desde qué altura hacer esta corrección para contrarrestar el incremento en la dosis de tóxicos condicionada por las variaciones de aclimatación del trabajador del ande. La recomendación en salud ocupacional, congruente con la norma fisiológica, es realizar el ajuste cuando la altura sea mayor a 2500

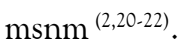

\section{Interacción de las variables}

Cuando en salud ocupacional se valora este conjunto de hechos, es necesario considerar otros factores importantes, como la naturaleza y grado de aclimatización del trabajador a la altura ${ }^{(2,4,16,19)}$, pues las variantes anatomofisiológicas resultantes de la aclimatación favorecen directamente su trabajo y permiten que se desempeñe en forma por demás óptima, independiente de la calidad geotópica de la labor. Además, considerar que indirectamente se expone al trabajador a mayor riesgo, ya que se utiliza en la actualidad valores límites de exposición recomendados para otras latitudes y realidades, casi siempre ubicadas en el llano y con menos horas diarias de trabajo.

El médico ocupacional en la altura debe tener clara la manera cómo el organismo del trabajador compensa los cambios de altitud y considerar que, en toxicología ocupacional, el esfuerzo físico no solo está en relación a la propia labor, sino que también depende directamente de la respuesta ventilatoria para el nivel de altitud donde esta se desarrolla y, por tanto, esa respuesta ventilatoria de compensación debe ser considerada como condicionante del aumento de la cantidad de tóxico que el trabajador absorbe ${ }^{(2,19,20-22)}$.

\section{A. Corrección de los valores límite umbral de exposición (TLV) para tóxicos inhalables en el trabajo de las grandes alturas}

Como premisa, no se requiere corrección para nivel de altitud, cuando se mide el tóxico para compararlo con un TLV, si ambas magnitudes son expresadas en condiciones volumétricas -partes por millón [ppm]-, porque ambos valores -el volumen del tóxico medido y los moles de oxígeno en el aire-son constantes a diferentes niveles de altitud, aún cuando el volumen de aire que contenga un mol de oxígeno varíe con la altura.

\section{a. Corrección del TLV por tiempo real de trabajo}

La corrección de los TLVs para la duración de la jornada laboral, cuando esta es mayor a 8 horas/día/5 días a la semana, se debe hacer llevándolos al tiempo real de trabajo según el método de Brief y Scala. Hoy, además, el Instituto para la Investigación en Salud y Seguridad del Trabajo del Canadá (IRSST) mantiene on line una herramienta útil que permite la conversión directa e inmediata de un TLV-TWA al tiempo requerido ${ }^{(24)}$.

Realizada esta corrección, aún es necesario ajustar el TLV para el nivel de altitud donde se ubica la labor.

\section{b. Corrección del TLV para la altura}

Para corregir los valores límite en los riesgos por inhalación de tóxicos en la altura, Knight ${ }^{(20)}$, Dummer ${ }^{(21)}$ y West ${ }^{(22)}$ proponen las siguientes alternativas:

- Corregir el valor del tóxico presente en el lugar de trabajo, correlacionándolo con la altura, o

- Aumentar el periodo de recuperación -descanso-, de manera tal que el organismo del trabajador tenga mayor tiempo para eliminar en forma natural el tóxico, lo que se consigue aumentando el tiempo libre de exposición entre jornada y jornada.
Para el primer caso, Dummer ${ }^{(21)}$ propone recalcular el valor obtenido en la medición en el ambiente de labor aplicando una regla de tres simple: multiplicar ese valor -ya corregido para el tiempo real de trabajo- por el valor de la PB de nivel del mar, y el resultado dividirlo entre el valor de PB del sitio de labor. Aquí, una digresión. Para realizar esta corrección, algunos higienistas usan densidad barométrica [densidad del aire $=1,3 \mathrm{~kg} / \mathrm{m}^{3}$ ] en lugar de PB y cuyo resultado difiere ligeramente. Pero, los fisiólogos ocupacionales están de acuerdo en usar presión barométrica (PB), a fin de estandarizar los resultados y visto que es más racional, pues es la presión barométrica y no la densidad del aire la que determina la difusión gaseosa a través de los alvéolos pulmonares $(20,25,26)$.

Si se usa la opción 2, y solamente cuando se trate de químicos a los que el organismo fisiológicamente es capaz de detoxificar, existe la posibilidad de aumentar el lapso de descanso entre jornada y jornada para ayudar así al organismo a liberarse del tóxico por mecanismos naturales. Sin embargo, este método no es válido para aquellos tóxicos que ingresan al organismo y no son eliminados, cuyo ejemplo representativo es la sílice, uno de los químicos más importantes en cuanto a riesgo para la salud asociado al trabajo minero. Se sabe que el polvo de sílice se acumula en el organismo y nunca más se elimina, pues es imposible sacarlo. Por tanto, en exposición a sílice, de ninguna manera considerar el método de aumento del periodo de recuperación. En ese caso, la recomendación es hacer un ajuste particular, que consiste en aumentar el tiempo de labor del trabajador en áreas limpias de la mina, donde el nivel de polvo esté por debajo del TLV ${ }^{(20)}$; y, cuanto más bajo, mejor.

c. Corrección del TLV para compensar el factor aumento de la ventilación

Se conoce en salud ocupacional que los TLVs son límites referenciales y 
empíricos. Por tanto, al valorar su eficacia, siempre considerarlos como tales y tener presente que podría haber una zona de penumbra donde un TLV deba recalcularse, máxime si la relación dosis-respuesta no es la esperada. Esta situación es relevante en el trabajo con tóxicos inhalables en la altura o cuando el trabajador se expone simultáneamente a varios tóxicos respiratorios.

En la gran altura, el organismo debe compensar la $\mathrm{PO} 2$ baja con mecanismos de aclimatación; de ellos, el aumento de la capacidad vital es el principal. Se ha determinado en diferentes grandes alturas que este incremento alcanza el $40 \%$. El hombre andino llega a este por ciento al corregir su ventilación por talla ${ }^{(3,6-9,12,15,16)}$, mientras que, en otros lugares, el aumento de la frecuencia ventilatoria es el determinante único ${ }^{(4)}$. La salud ocupacional debe confrontar el hecho que, debido a las modificaciones fisiológicas en la ventilación, la cantidad de un tóxico respiratorio presente en el aire alveolar, peso a peso y en unidad de tiempo, es mayor en la altura que a nivel del mar ${ }^{(5,20-22)}$.

Debido a estas circunstancias, y particularmente en este último caso, para aplicar técnicamente los TLV en el trabajo de la altura es necesario establecer una tercera corrección que compense los mayores volúmenes y capacidades pulmonares condicionadas por la mayor ventilación, $40 \%$ cuando se le corrige por talla $(2,3,11,16,17)$.

Por tanto, luego de realizar la corrección por tiempo de trabajo, del valor TLV a nivel del mar debería restarse ese $40 \%$ y el resultado recién ser considerado como valor TLV a aplicar para trabajo en la altura.

De esta manera, el procedimiento para la corrección compensatoria quedaría así:
Esta corrección contaría tanto para el trabajador nativo de la altura (aclimatado), quien ya tiene incorporado este peculiar mecanismo, como para el habitante del llano que asciende a trabajar en lapsos más o menos cortos [generalmente varían entre 1, 4, 7 o 14 días], para descender luego a su lugar de residencia (ascenso-descenso agudo) (figura 1).

Un acápite final. Por la naturaleza inexacta de la ciencia involucrada, se debe considerar que, al corregir estos límites de exposición, el utilizar una fórmula complicada puede resultar innecesario, si se tiene en cuenta que la recomendación primaria para el higienista y el ocupacionista debe ser realizar el control de cualquier riesgo aplicando el método primordial de eliminar el tóxico del ambiente de labor, lo que obviamente será suficiente para proteger al trabajador. Pero, si por la naturaleza de la operación no es posible, recién entonces aplicar fórmulas correctoras para reducir el valor límite de exposición. Y, si aún así el coste de ingeniería correctiva resultare oneroso, optar por cambiar 'corrección del valor del límite' por 'disminución del tiempo de exposición', acorde a la naturaleza del tóxico.

Solamente aplicando estos procedimientos, el ocupacionista estará seguro de obtener una reducción técnica y realista de los límites umbrales de exposición que asegure un mejor control de riesgos tóxico-respiratorios del trabajo en la altura.

\section{B. Corrección de los TLVs en la poliglobulia}

Si se valora el efecto de los tóxicos que se atesoran o se transportan en el eritrocito, la poliglobulia del hombre en el ande ${ }^{(11,12,16,17)}$ es tan importante como la fisiología respiratoria lo es para los tóxicos que ingresan por inhalación, pues para ese hecho no es igual tener un hematocrito de 39 que uno de 54 o

VALOR TLV $_{\text {ALTURA }}=$ TLV CORREGIDO POR TIEMPO - 40\% DEL VALOR TLV $_{\text {NVEL MAR }}$

60. Sin embargo, en salud ocupacional el campo toxicológico hemático de la altura aún no ha sido suficientemente explorado.

\section{Corrección de los TLVs en la exposición al ruido}

No existe necesidad de ajustar los valores permisibles de exposición al ruido en la altura, por cuanto la caída de la $\mathrm{PB}$ afecta por igual al sonómetro y al oído humano ${ }^{(20-22)}$.

\section{CONSIDERACIONES FINALES}

Al aplicar valores límites de exposición de los tóxicos respiratorios para el trabajo en las grandes alturas, es necesario hacerlo técnica y científicamente, realizando las correcciones necesarias para mayor tiempo de trabajo y altitud de labor, además de considerar las peculiaridades de la respuesta ventilatoria a esos niveles.

Los hechos revisados sugieren ampliar la investigación sobre aspectos respiratorios y hemáticos en el trabajador de la gran altura, a fin de establecer parámetros propios y seguros de exposición toxicológica.

\section{REFERENCIAS BIBLIOGRÁFICAS}

1. Hackett PH, Oelz O. The Lake Louise Consensus on the definition and quantification of altitude illness. En: Sutton JR, Coates G, Houston CS. Eds. Hypoxia and Mountain Medicine. Burlington. USA: Queen City Printers. 1992.

2. Åstrand P-O, Rodahl K. Fisiología del trabajo físico. Cáp. 17: Factores que afectan el desempeño físico. Bs. As., Argentina: Ed. Panamericana. 1995.

3. Gamboa AR. Ejercicio aeróbico. En: Monge CC y León-Velarde F. (Eds). El Reto fisiológico de vivir en los Andes. Cáp. 10. Lima: IFEA. UPCH. 2003.

4. Chiodi $\mathrm{H}$. Respiratory adaptations to chronic high altitude hypoxia. J Appl Physiol. 1957:10:81-7.

5. West JA. Aspectos sanitarios asociados al trabajo en las grandes alturas. ILO on line 2007. 37.10-37.4. http://www.ilo.org/encyclopaedia/?word\&nd=8571 00049\&nh=0 Consulta enero 2009.

6. Ramirez AV. Antropometria del trabajador minero de la altura. An Fac med. 2006;67(4):298-309.

7. Frisancho AR. Growth and morphology at high altitude. En: Baker PZ, Little MA (Eds). Man in the Andes. USA: Stroudsburg. 1976.

8. Greksa LP, Spiel-Vogel H, Cáceres E. Total lung capacity in young highlanders of Aymara ancestry. Am J Physiol Anthropol. 1994;94(4):477-86. 
9. Monge C. Acclimatization in the Andes: Historical confirmation of "Climatic Aggression" in the development of Andean man. USA: John Hopkins Univ Press. 1948.

10. Leon-Velarde F, Richalet S. Respiratory control in residents at high altitude: physiology and pathophysiology. High Altitude Medicine and Physiology. 2006; Tomo 7 (2).

11. Monge CC, León-Velarde F (Eds). El Reto fisiológico de vivir en los Andes. Lima: IFEA. UPCH. 2003.

12. Monge C. Aclimatación en los Andes. An Fac med. Lima. 1960.

13. Åstrand P-O. The respiratory activity in man exposed to prolonged hypoxia. Act Physiol Scand. 1959;30:343.

14. Lumbreras LG. El medio ambiente en los Andes. En: Monge CC y León-Velarde F. (Eds). El Reto fisiológico de vivir en los Andes. Cáp. 1. Lima: IFEA. UPCH. 2003.

15. Dollfus O. El reto del espacio andino. Instituto de Estudios Peruanos. Lima 1981.

16. Hurtado A. Animals at high altitude: resident man. En: Handbook of Physiology. American Physiological Society. Washington. 1964.

17. Hurtado A, Velasquez T, Reynafarge C, Lozano R, et al. Mechanism of natural acclimatization. Studies on the native resident of Morococha, Peru, at an altitude of 14900 feet. Report 56-1. School of Aviation Medicine USAF. Randolph Field. Texas. 1956

18. Hiba JC. Mineria y Trabajo Decente. Equipo técnico multidisciplinario para los países andinos. Lima: OIT. 2005.

19. Villena $\mathrm{CM}$ El ambiente en la altura. IBBA on line: www.ops.org.bo/altura/doc/htm. Consulta febrero 2010.

20. Knight D. Occupational TLV and mining work at high altitude. International SOS. London. 2007

21. Dummer W. Presión barométrica, reducción. OIT on line Capitulo 37. En: Enciclopedia de Salud y Seguridad en el trabajo: 2007;37:14-37.16. Disponible en: www.mtas.es/insht/EncOIT/Index.htm. Visita: febrero 2011

22. West JB. Health considerations for managing work at high altitude. OIT on line: 2007. http://www.ilo. org/encyclopaedia/?word\&nd=857100054\&nh= 0. Consulta enero 2010.

23. ACGIH TLVs. BEls. Threshold limits values for chemical substances and physical agents Biological Exposure Indices. Cincinnati. ACGIH. 2008. http:// www.ilo.org/encyclopaedia/?word\&nd=85710004 9\&nh=0 Consulta enero 2010

24. IRSST adjusted TLV - TWA calculation tool. 2007. IRSST on line. Consulta febrero 2010. http://www. irsst.qc.ca/en/ut_vema.htm;

25. Berger KI, Rom WN. Precautionary Note: Air Sampling. ILO on line: 2007. http://www.ilo.org/encycl opaedia/?d\&nd $=857100005 \&$ prevDoc $=8570000$ 09. Consulta 2010

26. Berger KI, Rom WN. Efectos fisiológicos de la reducción de la presión barométrica. ILO on line: 37.5 - 37.10, 2 007. Consulta dic 2009

27. http://www.ilo.org/encyclopaedia/?word\&nd=85 $7100049 \& n h=0$. Remmers JE, Mithoefer JC. The carbon monoxide diffusing capacity in permanent residents at high altitudes. Physiol. 1969;6:233-44.

28. Lindenbom RH, Palmes EA. Effect of reduced atmospheric pressure on a diffusional sampler. Am Ind Hyg Assoc J. 1983;44:105.

29. Lahiris S. Dynamic aspects of regulation of ventilation in man during acclimatization to high altitudes. Resp Physiol. 1972;16:245.

30. Ramírez AV. El tiempo circulatorio en la altura y a nivel del mar. Tesis de Bachiller en Medicina Humana. Universidad Nacional Mayor de San Marcos. Lima. Perú. 1969.

31. Edwards C, Heath D, Harris P, Castillo Y, Krüger H,
Arias-Stella J. The carotid body in animals at high altitude. J Pathol. 1971;104(4):231-8.

32. Arias-Stella J, Valcarcel J. Chief cells hyperplasia in the human carotid body at high altitude. Hum Pathol. 1976;7(4):361-73.

33. Saldaña MJ, Salem LE, Travesan R. High altitude hypoxia and chemodectomas. Hum Pathol. 1973;4(2):251-63.

34. Reynafarge B. Myoglobin content and enzymatic activity of muscle and altitude adaptation. J Appl Physiol. 1962;17:301.

35. Hurtado A, Rotta A, Merino C, Pons J. Studies of myohemoglobin at high altitudes. Amer J Sci. 1937;194:708.

36. Viault E. Sur la quantité d'oxygène contenue dans le sang des animaux des hautes plateaux de l'Amérique du sud. Comptes Rendus de Sciences de l'Academie de Sciences 1891;112:295-8.

37. Beall CM, Strohl KP, Blangero J, Willians-Blangero $S$, et al. Ventilation and hypoxic ventilatory response of Tibetan and Aymara high altitude natives. Am J Phys Anthropol. 1997;104(4):427-47.

Artículo recibido el 23 de agosto de 2010 y aceptado para publicación el 14 de febrero de 2011.

\section{Correspondencia:}

Augusto V. Ramírez, MD. $\mathrm{OH}$.

Dirección Salud Ocupacional.

Clínica Los Fresnos, Los Nogales 179, Cajamarca,

Perú

Teléfono: 051076364046 Ext. 244

Correo electrónico: augustovram@yahoo.es 